\title{
Short term load forecast of Kano zone using artificial intelligent techniques
}

\author{
Huzaimu Lawal Imam ${ }^{1}$, M. S. Gaya ${ }^{2}$, G. S. M. Galadanci ${ }^{3}$ \\ ${ }^{1,3}$ Department of Physics, Bayero University Kano, Nigeria \\ ${ }^{2}$ Department of Electrical Engineering, Kano University of Science and Technology, Wudil
}

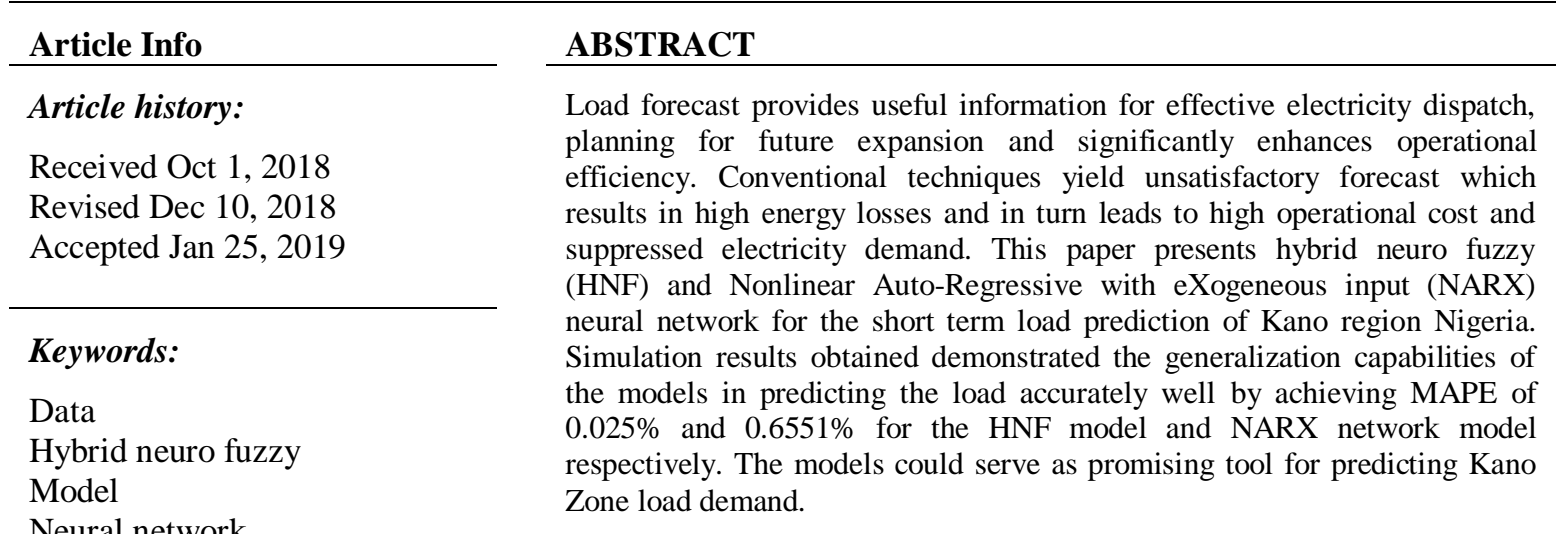

Copyright (ㅇ 2019 Institute of Advanced Engineering and Science. All rights reserved.

\section{Corresponding Author:}

G. S. M. Galadanci,

Department of Physics,

Bayero University Kano, Nigeria.

Email: ggaladanci.phy@buk.edu.ng

\section{INTRODUCTION}

Load forecast refers to the method utilized by energy supplying companies to predict the energy required to meet the demand and supply equilibrium. Load forecasting can be categorized as either a shortterm (which concerns a few hours prediction and mostly provides necessary information for the system management of day-to-day operations and power allocation) or medium-term (a few weeks up to a year prediction and this is used for the purpose of scheduling fuel supplies and unit maintenance) or long-term (over a year forecast used to provides electric utility company management with future expansion requirements, equipment purchases and performance improvement) [1].

It is significantly important to estimate hourly load and daily peak load [2] as these provide adequate information of load flows and helps in decision making. Appropriate implementation of the decision enhances network reliability, reduces frequent power outage and equipment failure. The accuracy of forecasting is of great significance for the system operation and energy management. However, traditional (both linear and nonlinear) forecasting tools when used yield unsatisfactory results which could be due to few samples of data sets or the data contained large amount of noise or non-stationary.

Intelligent techniques provide better alternative. These include various paradigm that uses artificial intelligence methods such as expert system [3], support vector machine (SVM) [4-5], Fuzzy Logic [6] and neural network. Expert system is a knowledge base method that builds load forecasting by imitating experience and human expertise. Nevertheless, the main issues with the knowledge based approach are need of large amount of time to acquire the required skills, errors may occur with knowledge base which in turn leads to wrong forecasting and cannot adapt to changing environment. SVM models are promising nonlinear approximation tools which usually produced good load forecasting results. SVM has much better generalization capability in practice, less risk of over fitting and effective extrapolation. 
Notwithstanding, choice of suitable kernel function, difficulty in understanding and interpreting of the final model are major limitations of support vector machine. Fuzzy logic is one of the most convenient and effective method of mapping an input space to an output space. The rule-based operation nature aids fuzzy logic in handing reasonable number of inputs and outputs. However, rules formation, choice of membership functions and adaptability are the main drawbacks of fuzzy logic. Hybrid neuro-fuzzy (HNF) and neural network are quite adaptive to changing environment, have the abilities of handling well rowdy data, uncertainties and producing reliable forecasting results [7-12].

The paper investigate the effectiveness of HNF and NARX- neural network in load forecasting. Several performance measures were used to evaluate the capabilities of the models in estimating the load. The organization of the paper is as follows: section 2 describes the materials and methods, section 3 presents simulation results and discussion while section 4 is the conclusion.

\section{RESEARCH METHOD}

\subsection{Zone Description}

Kano zone is one of the eleven electricity distribution zones in Nigeria with geographical coverage of $67,128 \mathrm{~km}^{2}$ comprising of Kano, Katsina and Jigawa States. The distribution capacity is over 2000MVA and the electricity comes from national grid supplied by Kumbotso 330/132/33KV, Dangora 132/33KV and Funtua 132/33KV transmission stations, with combined total installed capacity of 600MVA [13]. Part of the responsibilities of the zone are network coverage, provision of quality power supply and efficient marketing and customer services delivery. These can only be achieved with an appropriate load forecasting.

\subsection{NARX Neural Network}

The ability of neural network to learn and generalize make it an appropriate tool for approximating complex problems that are intractable [14]. Neural network has a built-in capability to adapt its synaptic weight to changes in the environment, this gives the network an edge over other nonlinear approximating techniques. NARX neural network belongs to a class of neural network termed as recurrent. NARX neural network requires a limited number of feedback as compared to other recurrent neural network. NARX network has a great computational abilities [15] and converges much faster with a better generalization. NARX neural network is expressed as follows:

$$
y(t)=\xi\left(u\left(t-n_{u}\right), \ldots . ., u(t-1), u(t), y\left(t-n_{y}\right), \ldots ., y(t-1)\right)
$$

where $\xi$ represents nonlinear function to be mapped, $u(t)$ and $y(t)$ depict the input and output of the network at time $t$ and $n_{u}$ and $n_{y}$ are the input and output order. NARX network is commonly configured as either parallel mode or series- parallel mode. Parallel NARX neural network is quite efficient especially in multi-step prediction and suitable for validation [16]. Parallel NARX network is given by:

$$
\tilde{y}_{p}(k+1)=\xi\left(u(k), \ldots . ., u(k-m+1) ; \tilde{y}_{p}(k), \ldots . ., \tilde{y}_{p}(k-n+1)\right)
$$

Although there is no established systematic method of determining the structure of the network. Nevertheless, appropriate network structure aids in producing an accurate forecasting results.

\subsection{Hybrid Neuro Fuzzy}

There are many ways of integrating neural network and fuzzy to form neuro-fuzzy system. The integration can be either concurrent or cooperative or hybrid neuro-fuzzy. Cooperative neuro-fuzzy as depicted in Figure 1 looks like a pre-processor in which the neural network (ANN) learning algorithm utilizes the training data set to obtain the fuzzy inference system (FIS) membership functions or fuzzy rules. Once the fuzzy inference system parameters are obtained, the neural network goes to the background. 


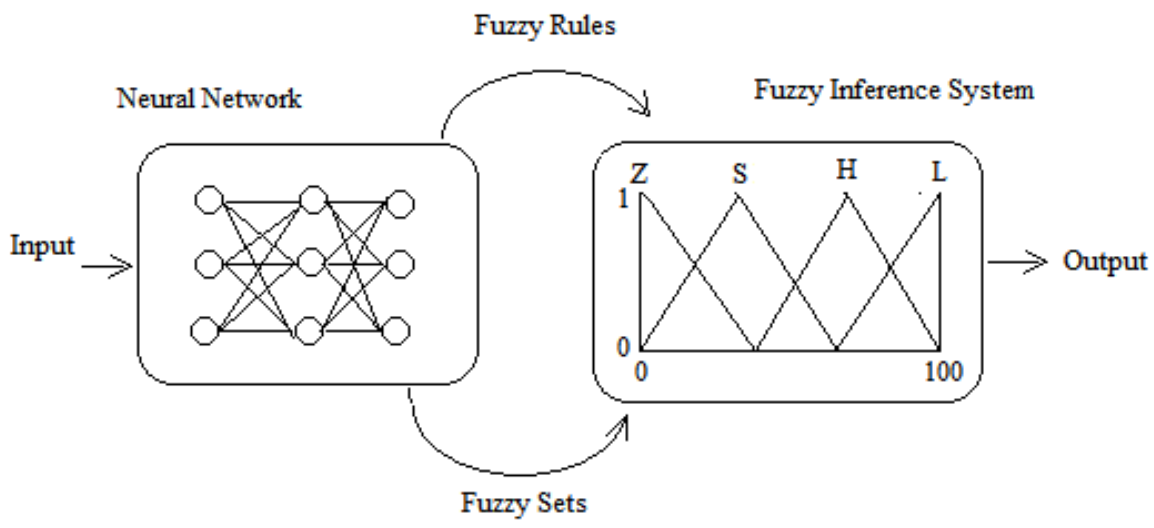

Figure 1. Cooperative neuro-fuzzy structure

In a concurrent nuero fuzzy shown in Figure 2, neural network aids the fuzzy system continuously (or vice versa) to obtain the required parameters especially if the input variables of the controller cannot be measured directly. This kind of combinations do not update the fuzzy system but only assists to enhance the performance of the overall system. Learning happen only in the neural network and the fuzzy system remains unchanged during this phase [17].

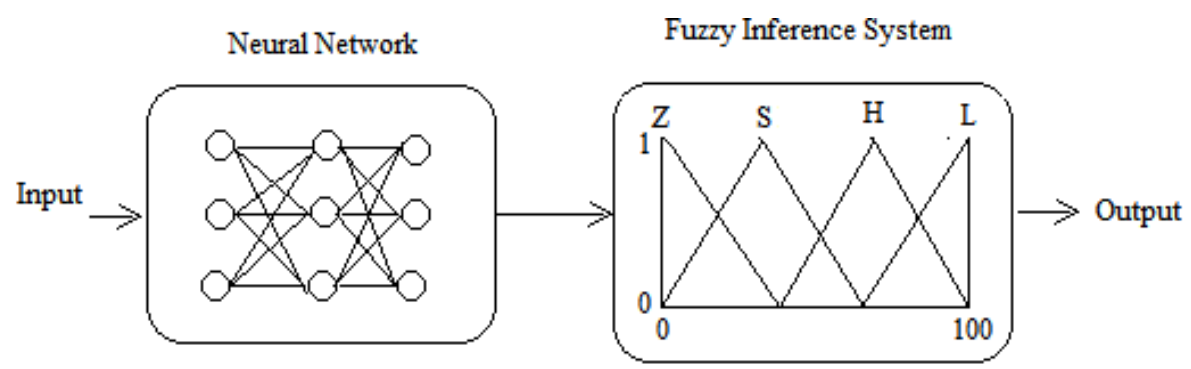

Figure 2. Architecture of Concurrent neuro fuzzy

In a hybrid neuro-fuzzy, the fuzzy system and neural network are one fully merged system. The fuzzy system utilizes the learning algorithm since it's depicted in a special neural network like structure [18]. Hybrid neuro fuzzy has supervised learning abilities as stated in [19-20]. HNF is made up of nodes, which depend on parameters that are updated based on certain learning algorithm to reduce error measure and links that always show the direction of information flow. The adaptive network received much attention from various fields as a result of its enticing features such as speedy convergence, accurate learning, ease of use, tolerating uncertainties and imprecise information [17]. The learning algorithm for the fuzzy inference system (FIS) parameters updating can be back-propagation or hybrid learning algorithm [21-23].

\subsection{Model Building}

A one day and half day hourly data were collected from Kano Electricity Distribution Company (KEDCO) to develop the models. To achieve reliable prediction, the data were preprocessed and randomly divided into training and testing data set. The training data set consisting of $80 \%$ in each case were used to build the forecasting models for one day and half day respectively. For testing the generalization capability of the models testing data set were utilized comprising of $20 \%$ for each model.

\subsubsection{Hybrid Neuro Fuzzy Model Structure}

Using the training data, the architecture of the HNF models for the one day and half day were realized. As illustrated in Figure 3, two gaussian membership functions were assigned to each inputs and this generated eight $\left(2^{3}\right)$ fuzzy rules. Each of these fuzzy rules yeilded one rule output and the aggregate of the rules produced the final output. 


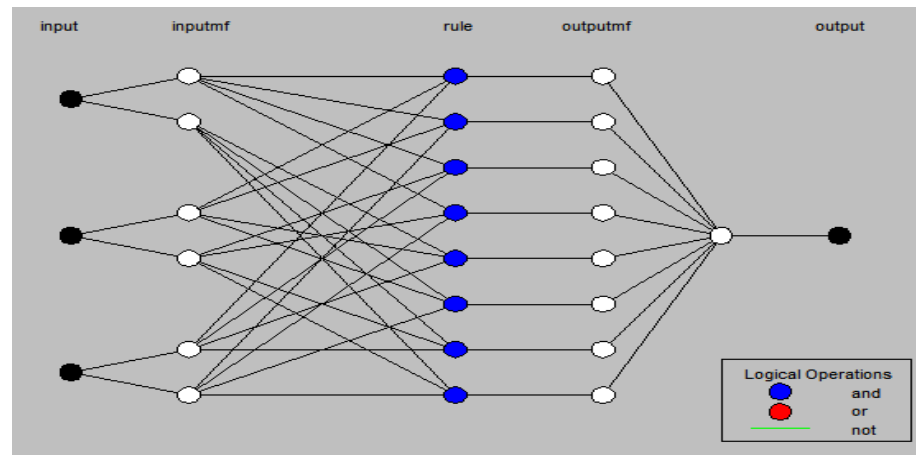

Figure 3. Hybrid Neuro Fuzzy Structure

\subsubsection{NARX Neural Network Structure}

Same training data set were used in realizing the structure of the NARX neural network, the structure was selected through trial and error since there is no established systematic method as shown in Figure 4. The hidden layer consisted of 10 neurons, [ $\left.\begin{array}{ll}0 & 1\end{array}\right]$ and [ 12 2] were used for input and output delay respectively.

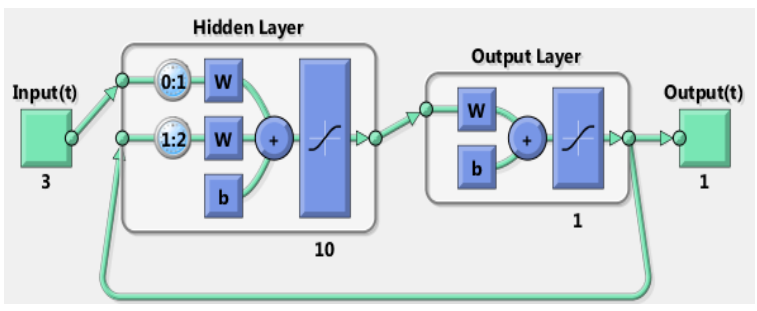

Figure 4. NARX Neural Network model Structure

\section{RESULTS AND ANALYSIS}

The performances of the proposed models during the training and testing phases for the one day and half day were illustrated in Figure 5, Figure 6, Figure 7 and Figure 8.

During the training phase the HNF and NARX neural network were able to achieved high accurate prediction as illustrated in Figure 5. However, in testing phase the performance of the ANFIS is slightly better than the NARX Neural network as dipcted in Figure 6. The accuracy of the models were evaluated using commonly used measures as Root Mean Square Error (RMSE) and Mean Absolute Percentage Error (MAPE). Table 1 presents the results obtained by the models during training and testing phases for the one day.

From the Figure 5 during the training phase the models demonstrated their capabilities in tracking well the trajectories of the observed data and achieved highly accurate forecasting with MAPE of 0.000024 and 0.019591 for the HNF and NARX NN respectively as illustrated in the Table 2.

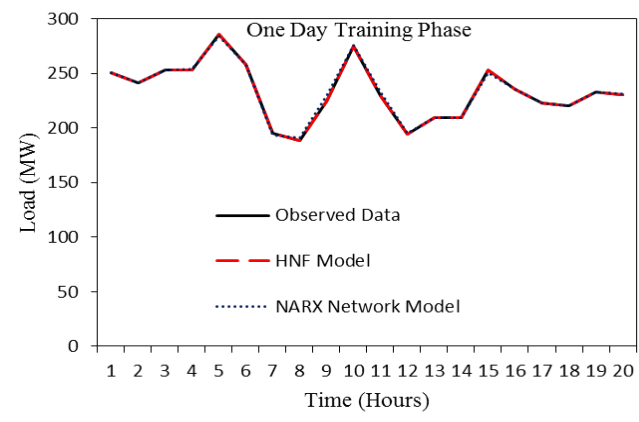

Figure 5. Models performance during training

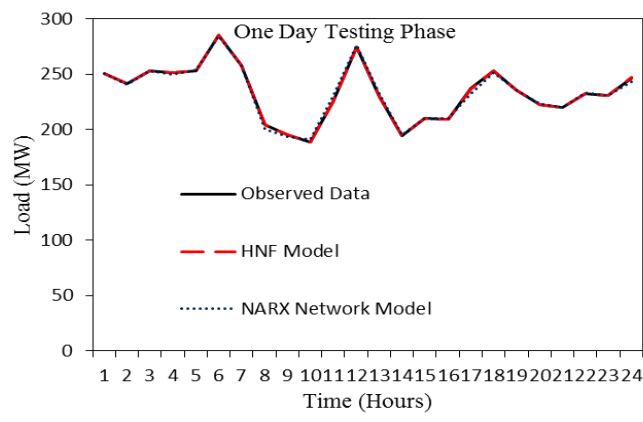

Figure 6. Models performance during testing 
Table 1. Full Day Model Performances

\begin{tabular}{ccccc}
\hline \multirow{2}{*}{ Model } & \multicolumn{2}{c}{ Training Phase } & \multicolumn{2}{c}{ Testing Phase } \\
& RMSE & MAPE $(\%)$ & RMSE & MAPE (\%) \\
\hline Hybrid Neuro Fuzzy & $7.3058 \mathrm{E}-11$ & $3.3288 \mathrm{E}-06$ & 0.2326 & 0.0250 \\
NARX Neural Network & $8.5474 \mathrm{E}-06$ & 0.4757 & 1.1145 & 0.6551 \\
\hline
\end{tabular}

The obtained results indicated that the models have accurately estimated well the observed data having achieved a MAPE of less than 10\% during the testing phase as illustrated in the Table 1. The generalization capabilities of the models are extremely accurate in line with a scale of judgement of forecast accuracy $[24,25]$.

The ranges of the data samples for the half day differ from the full day data set. The performances of the models for the half day during training and testing were illustrated in Figure 7 and Figure 8.

From the Figure 5 during the training phase the models demonstrated their capabilities in tracking well the trajectories of the observed data and achieved highly accurate forecasting with MAPE of 0.000024 and 0.019591 for the HNF and NARX NN respectively as illustrated in the Table 2.

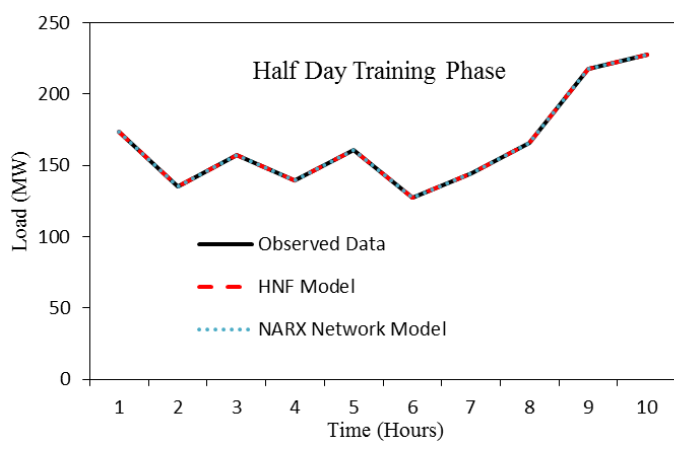

Figure 7. Half day models performance during training

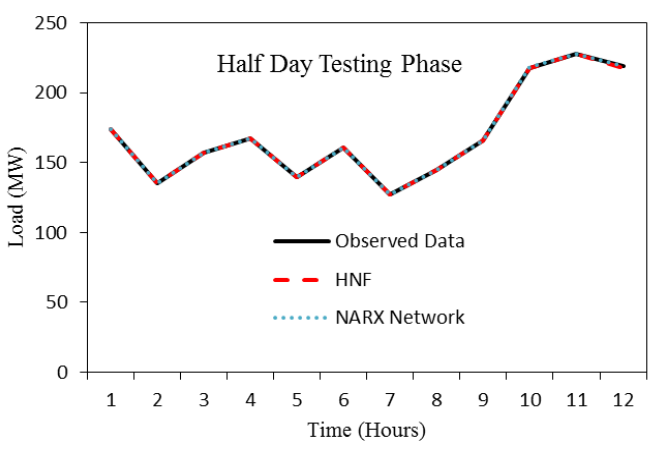

Figure 8. Half day models performance during testing

Table 2. Half Day Model Performance

\begin{tabular}{ccccc}
\hline \multirow{2}{*}{ Model } & \multicolumn{2}{c}{ Training Phase } & \multicolumn{2}{c}{ Testing Phase } \\
& RMSE & MAPE $(\%)$ & RMSE & MAPE (\%) \\
\hline Hybrid Neuro Fuzzy & 0.00011 & 0.00002 & 0.38332 & 0.05273 \\
NARX Neural Network & 0.06321 & 0.01926 & 0.00400 & 0.01753 \\
\hline
\end{tabular}

In the testing phase as depicted in Fig. 6 the models were able to efficiently estimate the observed data and the forecasting accuracy is quite high for both the models having achieved less than $10 \%$, although the NARX network is slightly better than the hybrid neuro fuzzy model, this could be connected to the choice of the suitable structure and generalization capability of NARX neural network.

\section{CONCLUSION}

The paper has presented hybrid neuro fuzzy and NARX network model for short term load forecasting of Kano Zone. The obtained results indicated the models achieved highly accurate forecasting of less than $10 \%$ in both the two cases (full day and half day). Apparently this proves their generalization capabilities especially when the data samples are few as compared to the traditional forecasting tools which mostly perform poorly for few data samples or non-stationary data samples. Smaller value of MAPE indicates the more accurate the forecasting model. The models may serve as an indispensable forecasting tools for the Kano Zone load demand.

\section{ACKNOWLEDGEMENT}

The authors wish to express their gratitude to KEDCO, Bayero University Kano and Kano University of Science and Technology, Wudil for their support 


\section{REFERENCES}

[1] Alfares H. K. and Nazeeruddin M., "Electric load forecasting: literature survey and classification of methods," International Journal of Systems Science, vol. 33, pp. 23-34, 2002.

[2] Espinoza M., et al., "Electric load forecasting using kernel- based modelling for nonlinear system identification," IEEE Control Systems Magazine, 2007.

[3] Rao M. S. S., et al., "An expert system approach to short term forecasting for reliance energy limited," Proceeedings of IEEE conference, 2006.

[4] M. Mohandes, "Support Vector Machines for Short-Term Electrical Load Forecasting," International Journal of Energy Research, vol. 26, pp. 335-345, 2002.

[5] Qiang S. and Pu Y., "Short term power load forecasting based on support vector machine and particle swarm optimization," Journal of Algorithms and Computational Technology, pp. 1-8.

[6] Luy M., et al., "Short term fuzzy load forecasting model using genetic fuzzy and ant-colony fuzzy knowledge base optimization," Applied Sciences, vol. 8, pp. 864, 2018.

[7] Yasmin N. S. A., et al., "Estimation of pH and MLSS using neural network," TELKOMNIKA Telecommunication Computing Electronics and Control, vol. 15, pp. 912-918, 2017.

[8] Gaya M. S., et al., "Estimation of Turbidity in Water Treatment Plant using Hammerstein-Wiener and Neural Network Technique," Indonesian Journal of Electrical Engineering and Computer Science, vol. 5, pp. 666-672, 2017.

[9] Yusuf Z., et al., "Neural network model development with soft computing techniques for membrance filtration process," International Journal of Electrical and Computer Engineering (IJECE), vol. 5, pp. 2614-2623, 2018.

[10] Gaya M. S., et al., "Compensation control of dissolved oxygen in an activated sludge system via hybrid neuro fuzzy technique," Procedia Manufacturing, vol. 2, pp. 307-312, 2015.

[11] N. A. Wahab, "ANFIS modelling of carbon and nitrogen removal in domestic wastewater treatment plant," Jurnal Teknologi, vol. 67, pp. 29-34, 2014.

[12] Gaya M. S., et al., "ANFIS direct inverse control of substrate in an activated sludge wastewater treatment system," Applied Mechanics and Materials, vol. 554, pp. 246-250, 2014.

[13] Gwamna J. I., "Strategically planning the strategic plan - KEDCO experience," PM World Journal, vol. 4, pp. 1-16, 2015.

[14] S. Haykin, "Neural network and learning machines," $3^{\text {rd }}$ Edition, Pearson Education, Inc., Upper Saddle River, New Jersey, 2009.

[15] Akhtar M., et al., "A dymanic neural network model for real-time prediction of zika epidemic in the America," 2018.

[16] Gaya M. S., et al., "Comparison of NARX neural network and classical modelling approaches," Applied Mechanics and Materials, vol. 554, pp. 360-365, 2014.

[17] Sumathi S. and Paneerselvam S., "Computational intelligence paradigms: theory \& applications using MATLAB," Taylor and Francis Group, LLC.

[18] Gaya M. S., "Neuro-Fuzzy Modelling and Neural Network Internal model Control of an Activated Sludge System," $\mathrm{PhD}$ Thesis, Universiti Teknologi Malaysia, 2014.

[19] Jang J., "ANFIS: Adaptive-network-based fuzzy inference system," Systems, Man and Cybernetics, IEEE Transactions, vol. 23, pp. 665-685.

[20] J. Jang, et al., "Neuro-fuzzy and soft computing: A Computational Approach to Learning and Machine Intelligence," New Jersey, Prentice Hall.

[21] J. S. R. Jang, "Input selection for ANFIS learning," Proceedings of IEEE 5th International Fuzzy Systems, New Orleans, LA: IEEE, pp. 1493-1499, 1996.

[22] Dasari M., et al., "GA-ANFIS PID compensated model reference adaptive control for BLDC motor," International Journal of Power Electronics and Drive System, vol. 10, pp. 265-276, 2019.

[23] Mujiarto, et al., "Colored object detection using 5 dof robot arm based adaptive neuro-fuzzy method," Indonesian Journal of Electrical Engineering and Computer Science, vol. 13, pp. 293-299, 2019.

[24] K. Lawrence, et al., "Fundamentals of forecasting using excel," New York, Industrial Press Inc, pp. 57-68, 2009.

[25] C. Lewis, "Industrial and business forecasting methods: A practical guide to exponential smoothing and curve fitting," California, Butterworth-Scientific, 1989. 